\title{
FIÈVRE JAUNE ET LE BESOIN CONSTANT DE SURVEILLANCE ÉPIDÉMIOLOGIQUE
}

\section{ARTICLE ORIGINAL}

GITIRANA, José Valdeci Almeida ${ }^{1}$

GITIRANA, José Valdeci Almeida. Fièvre jaune et le besoin constant de surveillance épidémiologique. Revista Científica Multidisciplinar Núcleo do Conhecimento. 04 année, Ed. 11, vol. 06, p. 05-15. novembre 2019. ISSN: 2448-0959, Lien d'accès: https://www.nucleodoconhecimento.com.br/sante/fievre-jaune

\section{RÉSUMÉ}

Objectif : dévoiler les facteurs qui justifient l'importance d'une surveillance épidémiologique efficace pour le contrôle de la fièvre jaune dans le pays. Méthode : recherche exploratoire, bibliographique, qualitative et descriptive. Résultats: les dernières enquêtes épidémiologiques indiquent que le nombre de cas de fièvre jaune sauvage enregistrés au Brésil est très variable, donc, s'il est considéré que pour chaque cas détectable il ya 10 autres cas d'évolution subclinique, les chiffres réels peut être beaucoup plus grande que celles enregistrées par les agences de santé. Conclusion : La fièvre jaune, en tant que maladie infectieuse grave, est une zoonose difficile à contrôler, car elle est capable de provoquer des épidémies imprévisibles dans les populations humaines, un exemple est le moment vécu par l'État du Minas Gerais, qui souffre d'une épidémie de maladie, motivée par des conditions de déséquilibre environnemental qui favorisent le développement et la prolifération des vecteurs, coopérant à l'apparition de centaines de décès dans les premiers mois de 2017 seulement.

\footnotetext{
${ }^{1}$ Spécialiste en analyse clinique, diplômé en pharmacie, actuellement universitaire en médecine.
} 
Mots-clés : contrôle, fièvre jaune, diagnostic, surveillance épidémiologique.

\section{INTRODUCTION}

La fièvre jaune est une maladie infectieuse grave, qui est un grave problème de santé publique dans la plupart des pays tropicaux, où les conditions environnementales favorisent le développement et la prolifération des vecteurs, causant des dizaines de cas de décès Annuellement. II peut se présenter sous des formes légères ou subcliniques et même des formes graves caractérisées par la triade composée d'ictère, d'hémorragies et d'insuffisance rénale aigue, avec un taux de mortalité, dans les cas graves, entre 20 et $50 \%$. ${ }^{24}$

L'agent étiologique de cette maladie est un arbovirus d'ARN appartenant à la famille Flaviviridae. Ce virus reste dans la nature dans un cycle sauvage, ayant des primates comme réservoir et les moustiques du genre Hemagogos comme vecteurs, et un autre urbain, dans lequel le réservoir est l'homme lui-même et a le moustique Aedes aegypti comme vecteur, dans ce cas précis la maladie est transmis de l'homme malade à I'homme unimmunisé par piqûre vectorielle. ${ }^{25}$

Sur le plan clinique, la période d'incubation est habituellement de trois à six jours. Cependant, des périodes plus longues ont déjà été décrites. Le spectre clinique de la maladie s'étend d'une condition bénigne, caractérisée par la maladie fébrile non spécifique, à une maladie fulminante caractérisée par le dysfonctionnement multiple d'organe, en particulier des hémorragie ${ }^{\varsigma} 1$. Ainsi, le patient peut subir une période asymptomatique, avec la fièvre suivante, le mal de tête, la douleur musculaire généralisée, la photophobie, les froids, l'ictère, et peut évoluer avec des conditions de saignement et l'échec rénal aigu ${ }^{2,3}$.

La fièvre jaune est une maladie de notification obligatoire et tous les cas suspects doivent être confirmés en laboratoire. Le diagnostic doit être corroboré par des données épidémiologiques, des manifestations cliniques décrites pour les différentes formes et des données de laboratoire. Cependant, un diagnostic plus précis est obtenu par la recherche sérologique des anticorps spécifiques et l'analyse histopathologique 
et immunohistochemical des spécimens de foie 4. Du point de vue pathopathique, des lésions peuvent être observées dans le foie, les reins, le cœur, la rate et les ganglions lymphatiques 1 , cependant, la lésion la plus importante de cette maladie se produit dans le foie au niveau des hépatocytes.

La fièvre jaune causant le virus est taxonomiquement classé famille Flaviviridae, genre Flavivirus. Le flavivirus est sphérique, enveloppant et mesure environ 40-50 nm. Son génome (ARN) se compose d'un ruban unique, linéaire, non segmenté, présentant un coefficient de sédimentation de 44S, contenant environ 100862 nucléotides, poids moléculaire de $4 \times 106 \mathrm{kd}$, Ont 10 gènes, qui encoderont 10 protéines : trois protéines C, M, E; et sept protéines non structurelles, NS1, NS2a, NS2b, NS3, NS4a, NS4b et NS5 ${ }^{5}$.

La protéine $C$ peut être en cappère viral. Les protéines $M$ et $E$ sont des constituants de l'enveloppe virale. La protéine $E$ est glycosylated et a des déterminants antigéniques importants, responsable principal de l'induire la réponse immunitaire et a également une valeur considérable pour la liaison du virus avec le récepteur de la membrane hôte. La protéine $M$ (non glycosylated) est le résultat du clivage de la protéine pré-M (glycosilated) 1.

Cependant, il y a des études qui ont également souligné l'importance des protéines NS1 et NS3 dans la réponse immunitaire de l'hôte. Le premier a la capacité d'activer le complément et d'induire la lyse des cellules infectées, tandis que NS3 est l'une des principales cibles de l'attaque cytotoxique des lymphocytes T 6.

Après pénétration des particules virales dans le cytoplasme et le dénudement, l'ARN se réplique dans les régions périnucléaires, médiées par un $A R N$ de polarité négative. La maturation des visrions se produit dans les membranes intracellulaires, avec le virus poussant de l'appareil Golgi ou le réticulum endoplasmique, et deux types distincts de particules virales peuvent être définis: les virus associés aux cellules et aux particules virales extracellulaires. ${ }^{26}$ 
Les virus appartenant à la famille des Flaviviridae ont la capacité de se répliquer chez les moustiques et les primates, y compris l'homme. Ils ont une grande adaptabilité, ce qui implique de dire que cette caractéristique a une relation directe dans le maintien de ces virus dans la nature. ${ }^{27}$

D'un critère éminemment épidémiologique, on peut définir le virus de la fièvre jaune comme un arbovirus dont le cycle primaire implique principalement les moustiques forestiers et les espèces de primates non humains, en particulier Callitrichidae et Cebidae, chez les primates néotropicaux, la plupart des espèces d'Amérique du Sud lorsqu'elles sont infectées, en général, présentent une évolution mortelle, un fait qui n'est pas observé chez les espèces africaines. De même, des espèces comme les oiseaux, les rongeurs, les marsupiaux, les carnivores, les amphibiens et les reptiles sont généralement résistantes au virus de la fièvre jaune 7 .

Les moustiques impliqués comme vecteurs de la fièvre jaune sont anthropophiles de l'activité diurne dans la cime des arbres, dans l'environnement sauvage et donc l'infection humaine est accidentelle et conséquente à la pénétration humaine dans l'endroit où la zoonose se produit. En milieu urbain, il est nécessaire la présence de vecteurs vivant dans la maison ou péridomique de l'homme urbain 1.

Plusieurs espèces vectorielles de cette pathologie ont été étudiées au fil des ans et peuvent varier selon la région étudiée, parmi elles nous pouvons mentionner : Haemagogos janthimomys, Haemagogus albomaculatus et leucocelaenus, $\mathrm{Hg}$. janthinomys, Sabethes chloropterus et Sabethes soperi, Aedes abopictus et Aedes aegypti, entre autres. ${ }^{28}$

Du point de vue de la transmission, l'homme joue un rôle important dans la propagation du virus dans des zones où il ne se produit pas naturellement, mais qui ont des vecteurs et des singes, qui font partie du cycle de transmission de la maladie. En outre, les changements dans la forêt, habituellement causés par l'ouverture des routes, l'exploitation forestière et l'activité agricole, exposent accidentellement les individus non vaccinés aux vecteurs d'invertébrés sauvages, étant l'une des principales formes de maladie dans l'Amazonie brésilienne ${ }^{3,4}$. 
Il convient de rappeler que, même s'il n'est pas répandu dans notre pays, une autre forme de maintenance du virus dans la nature peut se produire dans les zones urbaines, avec Aedes aegypti étant le vecteur impliqué et l'homme le seul hôte. La forme urbaine de la fièvre jaune a été éradiquée du Brésil en 1942. Cependant des études ont montré la présence d'Aedes aegypti dans environ 21 États brésiliens ${ }^{7}$.

Par conséquent, un patient virémique d'une zone endémique de fièvre jaune sauvage peut, associé aux densités élevées d'Aedes aegypti, constituer un stimulus potentiel pour la réurbanisation de la fièvre jaune, qui aurait de graves conséquences en particulier pour la population précédemment immunisée ${ }^{7,4}$.

Des études ont soulevé l'hypothèse d'une participation possible d'Aedes albopictus au cycle de transmission de la maladie, dont la capacité de transmettre le virus a déjà été démontrée en laboratoir ${ }^{e 1,8,9}$. Parce que cet agent peut se reproduire dans les milieux ruraux, périurbains et urbains, il peut potentiellement servir de pont entre le cycle sauvage et urbain de la fièvre jaune. En Afrique, plusieurs espèces du genre Aedes, comme Aedes furcifer, Aedes taylori et Aedes luteocephalus, sont des vecteurs urbains aussi importants qu'Aedes aegypti 5.

La réponse à l'infection à amaralique est large et variable, dans cette perspective, on estime qu'environ $90 \%$ des cas avec l'expression clinique sont des formes classifiées comme douces et oligosymptomatiques, c'est-à-dire présentant peu de symptômes et que seulement $10 \%$ sont des formes Grave. En outre, il y a des personnes qui développent des conditions asymptomatiques 8 .

De telles formes asymptomatiques, ainsi que des cas subcliniques et des formes bénignes de la maladie sont souvent perceptibles chez les enfants de bas âge dont les mères ont été vaccinées et leur ont transmis des anticorps maternels IgG 9. D'autre part, il ya des individus qui développent des formes plus graves de la maladie, comme les personnes qui n'ont jamais été vaccinés et, par conséquent, sont complètement impuissants à la maladie. Ces individus, à leur tour, développent des conditions cliniques graves et extrêmement graves de la maladie. 
Il est à noter que dans les formes bénignes et modérées de la symptomatologie de la maladie est indifférenciée et non caractéristique, c'est-à-dire qu'elle est confondue avec de nombreuses maladies courantes dans les zones endémiques, telles que le paludisme, l'hépatite virale, la fièvre typhoïde, parmi 10 autre $^{\text {re }}$.

En général, les symptômes sous forme légère sont limités à des maux de tête et de l'asthérie, accompagnés de febrilcula ou de fièvre modérée. II évolue jusqu'à deux jours, après la fin de ceux-ci le patient recompose sans montrer de séquelles. Dans sa forme modérée, le patient en plus d'être en mesure de présenter ces symptômes peut même être accompagné de nausées avec l'absence de vomissements, en plus de myalgies et d'arthralgies qui ne dérangent même pas le patient ou empêcher sa locomotion.

Les maux de tête durent généralement plus longtemps. La fièvre ne se termine qu'avec l'utilisation de sables antithermiques déjà plus persistants. Au moins une des indications classiques de la maladie est accompagnée de cette image symptomatique : jaunisse ou oligurie/anurie et hématoyhe (vomissement noir)8. La durée d'un tel état est de deux à trois jours et le rétablissement fait partie intégrante. L'image évolue en deux périodes, avec une phase de rémission entre eux. II convient toutefois de souligner qu'il n'est pas toujours possible de séparer ces phases. ${ }^{8}$

La symptomatologie de la forme classique de la fièvre jaune est grave et caractérisée par un début brusque, après une période d'incubation moyenne de 6 jours (période allant de la morsure infectieuse à l'apparition de la manifestation des symptômes) et qui peut durer environ deux semaines. Au début, il ya une forte fièvre, dans laquelle le patient déclare avoir senti bien et terminé ses occupations quelques heures avant 8 . La fièvre n'est pas accompagnée d'une augmentation du pouls ${ }^{1} 1$.

Inversement, il est fréquent d'observer une forte fièvre avec une baisse de pulsation, un tel signe est connu sous le nom de signe faget ${ }^{1} 2$. Peu de temps après le commencement de la fièvre, le mal de tête holocrânien d'une telle intensité se manifeste que le patient se sent souvent le besoin de demander l'utilisation des analgésiques ${ }^{1} 3$. 
Ces situations accompagnent des douleurs musculaires diffuses, en particulier dans le dos, où ils sont généralement présents. Asthése, prostration et nausées concluent cette image qui évolue de 2 à 3 jours et correspond à la période prodromic 8 .

Dans plusieurs patients, évoluant à la période infectieuse, il y a une phase d'amélioration qui est caractérisée par un sentiment de soulagement et de guérison immédiate de la part du patient, parce que la basse fièvre ou bien cesse, la douleur de muscle diminue et le mal de tête passe à un état Supportable.

Cette période dure de 12 à 48 heures et, après cela, contrairement aux individus qui développent les formes douces et modérées qui guérissent réellement, l'état de ces patients s'aggrave soudainement, avec l'aggravation de tous les symptômes précédemment présentés, avec le l'émergence de 11 autre $^{\mathrm{r}} \mathrm{s}$. Ce signal est le début de la période d'intoxication, toxémique ou "phase de localisation", car dans cette période le virus devient circulaire dans le sang pour être situé uniquement dans le foie. Dans lui, la nausée s'intensifie et des vomissements apparaissent brusquement. Ces symptômes sont l'onset sont manger et puis largement hémorragique ${ }^{12}$.

Le vomissement est la couleur de tache de café si le sang a souffert la réaction du jus gastrique, ou largement hémorragique si le saignement est récent ou intense. II est démontré que d'autres manifestations hémorragiques sont couramment trouvées. Les hémorragies les plus présentes sont des tissus tégumentaires, des gencives et de l'oreille ${ }^{14}$. Dans le tractus gastro-intestinal, melena peut se produire, quand I'hémorragie d'estomac est large ou quand elle présente la manifestation du saignement d'entrailles ${ }^{15}$.

Accompagnant ou même précédent saignement, il ya la présence de thrombopenia, souvent si intense qu'il peut atteindre moins de $20.000 / \mathrm{mm} 3$ de sang. II est intéressant de montrer que certains patients, même avec de tels niveaux de plaquettes, ne saignent pas. Alors que d'autres avec des taux entre 50.000 et 100.000 plaquettes manifestent des saignements exorbitants. On remarque que le degré d'intensité de I'hémorragie n'est pas toujours interconnecté avec le nombre de plaquettes ${ }^{14}$. 
Parmi ces symptômes, l'ictère, l'existence de la couleur jaunâtre sur la peau et les yeux sclérosés 14 apparaît. La jaunisse est, en bref, du type verdainien et est due au fait de l'augmentation de la fraction directe 8. Autour du 5ème au 7ème jour de la période d'état, l'échec rénal est installé, qui présente d'abord dû au volume urinaire diminué et, si non traité rapidement peut déclencher l'anurie et l'arrêt complet de la diurèse due à la nécrose tubulaire aigue largement répandue qui est installée. C'est le moment où les décès 11,12 se produisent le plus so ${ }^{\text {uvent }}$.

Ceux qui survivent récupèrent lentement, mais totalement et sans séquelles. Pendant la convalescence, l'indisposition, l'asthérie et la douleur musculaire s'étendent habituellement jusqu'à deux semaines.

II convient de noter qu'en Afrique, l'apparition de formes fulminantes sont souvent signalées, qui se caractérisent par une évolution allant jusqu'à trois jours, résultant d'une insuffisance rénale et, presque toujours, sans la présence d'ictère scinde ou même d'altérations hépatiques ${ }^{1} 6$.

À l'autre extrême, de rares cas de décès tardif dû à la fièvre jaune peuvent être mis en évidence, des cas qui sont associés à des lésions cardiaques tardives ${ }^{17}$. Enfin, on ne peut pas souligner que dans les cas où l'ictère est brusque et les niveaux de bilirubine du sérum sont très élevés encéphalopathie est fréquent, étant un signe de pronostic pauvre. Cependant, la plupart des patients progressent à la mort soit par insuffisance hépatique ou due au saignement, qui sont souvent incontrôlables.

L'examen spécifique pour le diagnostic définitif de la fièvre jaune est l'isolement du virus, également appelé examen de culture. Grâce à cet examen, il est possible de détecter les antigènes viraux et l'ARN viral 9. En outre, il est possible de diagnostiquer la maladie par l'utilisation de méthodes sérologiques, telles que la posologie d'anticorps spécifiques par la méthode ELISA MAC en capturant IgM dans un test enzymatique ou une conversion sérologique dans les tests d'inhibition de la hémaglutination $(\mathrm{IH})^{1} 8$. 
Dans cette perspective, l'isolement du virus peut se faire en combinant différents systèmes, comme dans le cas de l'isolement du virus, ce ne sera possible qu'après l'inoculation de l'échantillon suspect, lorsque des preuves de réplication virale sont obtenues autour de la 5 e à la $7 e$ - jour de culture. Ainsi, une fois séparé, l'échantillon viral peut être identifié dans des essais indirects d'immunofluorescence, utilisant des anticorps monoclonaux ou alternativement par des essais de fixation de complément 16.

D'autre part, les méthodes sérologiques qui indiquent l'IgM spécifique, comme avec le cas de Mac ELISA, peuvent donner un diagnostic présomptif agile avec un échantillon sérologique, s'il est recueilli du patient du 5ème jour de la maladie ${ }^{19}$. Ainsi, la manifestation de l'IgM peut être le résultat d'une infection récente (2-3 mois) ou actuelle (actuelle), pour cette raison l'importance d'avoir l'histoire épidémiologique et clinique complète pour l'interprétation du résultat de laboratoire. Il est important de souligner que la vaccination antiamarille peut favoriser la formation d'IgM et, par conséquent, il est extrêmement important de connaître les antécédents de vaccination du patient.

Comme il n'existe pas encore de médicament spécifique et efficace pour le traitement de cette maladie, les tests qui diagnostiquent comme positif pour l'infection prennent environ une semaine. Le traitement de soutien doit être commencé immédiatement, et I'hospitalisation du patient avec des formes graves dans les hôpitaux avec une bonne préparation de l'infrastructure pour toute maladie et de préférence dans I'USI est catégorique, car il ya un besoin d'un certain nombre de procédures qui ne délibèrent que plus facilement dans ces unités ${ }^{1} 2$.

Cependant, le traitement médicamenteux ne devrait être concentré que pour combattre les symptômes, de donc le médicament à prescrire dépend des manifestations cliniques du patient, mais il est d'usage d'utiliser des antithethermaux et des analgésiques aux doses couramment indiquées, allant de en fonction de facteurs tels que l'âge et le poids. 
Il convient de souligner la contre-indication de l'utilisation de médicaments qui ont de l'acide acétylsalicylique ou de leurs dérivés dans leur formule, car il peut aggraver les phénomènes de saignement qui peuvent être associés à l'évolution de la maladie ${ }^{8,1} 1$.

Avec le vomissement, les médicaments de contrôle tels que le metoclopramide sont communs. En outre, il est recommandé l'utilisation de médicaments pour préserver la muqueuse gastrique, appelé anti-H2, comme la cimétidine et la ranitidine, avec ceuxci comme un exemple, qui sont extrêmement utiles pour prévenir les saignements gastriques. Ces saignements peuvent être consolidés comme l'une des complications les plus mortelles de la fièvre jaune. Quand l'échec rénal est démontré par l'oligurie il est important que les drogues diurétiques devraient être prescrites ${ }^{19}$.

On ne peut pas souligner que l'évaluation du patient doit être constante et comprend des actions telles que l'étude des signes vitaux, la diurèse, et la demande d'au moins les tests suivants: compte sanguin, plaquettes, résumé de l'urine et la vérification des fonctions hépatiques ( dosage d'aminotransferases, de bilirubine et de gamme GT) et rénaux (dosage d'urée et de créatinine, et surveillance de l'équilibre de l'eau)'19. Ainsi, étant donné les conditions dans lesquelles les patients ont l'échec rénal installé et ne répond pas aux diurétiques communs il est important d'indiquer l'hémodialyse ou la dialyse péritonéale, selon l'évolution du patient et considérant l'aggravation de la condition.

\section{MÉTHODE}

La fièvre jaune a été dans le passé comme un grand martyre pour la population brésilienne et même aujourd'hui, malgré l'existence de l'antiamarillique valin, il est source de préoccupation. Surtout, en raison de la sévérité qu'il peut atteindre et peut même évoluer à la mort. II s'agit d'une maladie infectieuse grave, qui est un grave problème de santé publique dans de nombreux pays tropicaux, où les conditions environnementales favorisent le développement et la reproduction des vecteurs. Son agent étiologique est un arbovirus à ARN appartenant à la famille Flaviviridae. ${ }^{4}$ Dans le cadre de ce travail, un point de vue global est lancé sur les différentes façons de 
faire face à ce grand problème de santé publique et sur l'importance de son contrôle grâce à un système efficace de surveillance de la fièvre jaune.

Ainsi, cette recherche a été réalisée qualitativement 22 car elle aborde la fièvre jaune, soulignant la nécessité d'une surveillance épidémiologique constante. Dans cette perspective, cet article est présenté sous forme descriptive, sur la base du regard théorique utilisé dans la recherche, rapportant les principales caractéristiques de la pathologie, qui raffermissent la nécessité d'une surveillance dans le pays par des actions qui mettre l'accent sur la prévention.

\section{RÉSULTATS}

La fièvre jaune est une maladie de notification obligatoire et, en tant que tel, tout cas de suspicion doit être immédiatement communiqué à l'autorité sanitaire locale, étatique ou nationale, qui à son tour a le devoir de signaler l'affaire aux organismes internationau ${ }^{\times 2} 0$.

Si elle est confirmée ou réglée, la notification de l'affaire est transmise à l'autorité nationale, qui à son tour confirme ou rejette l'assignation à comparaître à l'autorité sanitaire internationale. Cela devrait toujours être fait pour accélérer les mesures préventives et prévenir d'éventuelles flambées ${ }^{2} 1$.

Il est important de souligner que la méthode la plus efficace pour prévenir l'apparition de la fièvre jaune est toujours la vaccination avec l'échantillon 17D. De ce point de vue, il est mis en garde que toutes les personnes en bonne santé de plus de six mois, qui sont exposées ou exposées à une contamination possible par l'infection 8,10,16, sont correctement vaccinées.

Une seule dose du vaccin protège l'individu pendant au moins 10 ans, période au cours de laquelle la revaccination est recommandée. Cependant, il convient de souligner que certaines études ont montré que les personnes vaccinées une seule fois et vivant loin des zones à risque indiquent neutraliser les anticorps pendant 35 ans, ce qui dénote la fiabilité et l'efficacité du vaccin ${ }^{1} 6$. 
Compte tenu du fait que le vaccin est produit avec le virus vivant atténué, il n'est pas conseillé que les personnes immunodéficientes au risque d'inversion de la virulence chez un hôte souffrant de dépression du système immunitaire soient vaccinées ${ }^{2} 9$.

Les patients atteints de cancer, de sida/SIDA et utilisant des médicaments qui diminuent la réponse immunitaire de l'organisme ne devraient pas être vaccinés, sauf dans les cas spécifiquement prédéterminés par l'autorité médicale 8.

Par conséquent, outre la vaccination, la lutte contre les vecteurs et l'utilisation de la protection individuelle sont des formes de prévention de la fièvre jaune, mais il ne faut pas la perdre compte tenu du fait que la lutte contre les vecteurs forestiers est fondamentalement irréalisable, de sorte que la lutte contre le vecteur urbain devient relativement facile ${ }^{2} 9$.

\section{DISCUSSION}

Compte tenu de la récente flambée de fièvre jaune sauvage qui sévit dans la zone rurale de l'État du Minas Gerais et se propage dans tout le pays, il est indéniable de souligner qu'il est du devoir de la coordination du Programme de surveillance et de contrôle de la fièvre jaune (PVCFA) de trouver des solutions pour améliorer et sophistiqué la surveillance de la maladie sur le territoire national, parmi les actions qui englobent ce devoir sont les suivantes: définir les zones à risque, permettre aux laboratoires d'avoir les moyens d'identifier les cas de la maladie, promouvoir des actions d'éducation sanitaire afin de sensibiliser, entre autres, à la nécessité de la prévention. Toutefois, certaines mesures 23 devraient être mises en œœud afin de rendre la surveillance épidémiologique au Brésil par rapport à la fièvre jaune plus efficace, telles que :

- Adopter une définition de cas plus ouverte que celle conseillée par l'Organisation panaméricaine de la santé (OPS), introduisant de préférence une approche syndromic, dans le but d'accroître la réceptibilité et l'utilité du système ${ }^{20}$; 
- Améliorer la qualification de l'information afin d'accroître la commodité du système dans l'analyse et la détection des tendances historiques ${ }^{2} 0$;

- Faire évoluer le système de surveillance des épizootiques et entomologiques comme axes primordiaux de détection précoce de la circulation virale au Brésil, en particulier dans les zones où la population résidente n'est pas encore vaccinée ${ }^{2} 0$;

- Intégrer les connaissances, les nouvelles techniques et technologies pour réaliser les prémisses du programme, telles que l'utilisation de l'information géographique pour détecter les tendances possibles de la distribution et de la dispersion virales et vérifier la corrélation avec les données environnementales, entomologique et démographique, en mettant l'accent sur l'élaboration d'un modèle capable de calculer les zones à risque de fièvre jaune ${ }^{2} 0$.

Il convient de noter que, bien que la dernière manifestation de la fièvre jaune urbaine ait été documentée il y a plus de 60 (soixante) ans dans le pays, la possibilité d'une réapparition ne peut être écartée. Surtout lorsque des facteurs potentiellement favorables peuvent être facilement identifiés ${ }^{1} 4$.

L'un de ces facteurs est précisément la dispersion d'Aedes aegypti dans des milliers de municipalités, qui est la nécessité de nouvelles possibilités pour la santé publique. Surtout l'ordre sanitaire ${ }^{23}$.

En outre, on ne peut manquer de souligner le fait que le maintien de la prolifération de la fièvre jaune sauvage est une intention pour la santé de la population. Principalement en raison de la capacité épidémique qu'il peut assumer dans les populations urbaines qui ont des taux inférieurs à $50 \%$ de la couverture vaccinale ${ }^{22} 3$.

En bref, la combinaison de ces idées indique que la surveillance de la fièvre jaune devrait travailler côte à côte avec la surveillance des cas humains, la surveillance des épizootiques chez les primates non humains et la surveillance entologique, en vue d'accroître l'efficacité pour plus de la circulation virale ${ }^{2} 3$. 
Pourquoi tout cas soupçonné de fièvre jaune doit être transmis immédiatement aux organes appropriés et, après notification, une enquête immédiate doit être menée qui ne doit pas dépasser la période maximale pouvant aller jusqu'à 24 heures après l'assignation, le résultat devrait être divulgué dans les 60 jours.

De ce point de vue, les actions de surveillance épidémiologique sont des actions face à des cas humains présumés :

- Détecter autant de cas suspects que possible (symptomatiques et asymptomatiques) et les aviser d'augmenter l'évaluation de la population à risque;

- Trouver des cas d'épizootiques sur le territoire afin d'identifier la zone à risque;

- Guider les actions de lutte contre l'épidémie (vaccination et lutte contre le vecteur urbain);

- Mettre en évidence les résultats.

Comme mentionné, la maladie intègre un large éventail de diagnostics différentiels avec des maladies telles que le paludisme, l'hépatite, la leptospirose et d'autres maladies de l'ichthyse et/ou hémorragiques, qui se ferment dans un pourcentage élevé de cas non pris en compte par les dossiers des systèmes de surveillance.

Dans ce cadre, le PVCFA recommande l'approche syndromic en matière de surveillance par l'utilisation d'un formulaire de calcul et d'entretien unique par le biais d'essais en laboratoire, comme moyen de déterminer l'opportunité et d'accroître l'efficacité, l'adhésion scientifique et la caractérisation du système national de surveillance épidémiologique, visant à postuler l'éveil méthodique et constant du syndrome fébrile ichthyique et/ou hémorragique dans les unités de service sentinelles, en priorisant la mesure méthodique et constante des cas soupçonnés ${ }^{2} 3$.

La surveillance entomologique est également un moyen de lutter contre la maladie, en particulier par la saisie de vecteurs sauvages dans les endroits où les épizootiques et/ou les cas humains se produisent. 
En outre, le renforcement de la capacité de laboratoire est essentiel, surtout lorsqu'il est considéré que le principe de ce sous-composant est d'améliorer l'efficacité de laboratoire pour l'identification précoce du virus de la fièvre jaune chez les primates qui ne êtres humains et êtres humains eux-mêmes ${ }^{24}$.

Avec cela, la base pour le diagnostic est nécessaire avec un accent sur la recherche sur le virus dans les vecteurs potentiels, ajouté à la construction de données pour la détection des espèces de cisicides confinés dans les zones de recherche. Il est à noter que, de nos jours, le réseau de laboratoires provient de laboratoires centraux (LACEN), en mettant l'accent sur l'Institut Adolfo Lutz, aussi distingué régional et I'Institut Evandro Chagas que le laboratoire de référence national ${ }^{3} 0$.

L'éducation sanitaire est un point à mentionner, surtout lorsqu'on considère qu'il s'agit d'un mécanisme important et qu'elle devrait occuper une place centrale dans les actions de surveillance épidémiologique, car il est nécessaire que la population comprenne en fait que la maladie doit être de modifier sa conduite vers la prévention $9,10,20,21$.

Enfin, on ne peut manquer de mentionner les conceptions de la surveillance de la fièvre jaune au Brésil. Dans cette perspective, il a été que l'une des fonctions de la surveillance épidémiologique est de surveiller l'état de santé des populations pour aider les politiques publiques afin de fournir des endroits sûrs et sains 15 , compte tenu de cela, il cherche à réaliser le suivi fièvre jaune d'un biais créatif, afin d'atteindre tous les nombreux espaces de flambées de prolifération de la maladie, qui, une fois détecté et cartographié, deviendra des territoires d'intervention ${ }^{1} 3$.

Par conséquent, les mesures en ce sens devraient être dirigées pour mieux clarifier les facteurs de risque écologiques et environnementaux qui articulent la circulation du virus.

Ainsi, l'obtention de données issues de la mise en œuvre de nouveaux outils et de nouvelles perspectives devrait fournir des informations précises supplémentaires afin que des lignes directrices d'action viables puissent être postulées, qui sont basées sur 
des bases d'analyse fermes et précises et l'interprétation, afin qu'ils puissent générer de nouvelles recommandations de mesures de prévention et de contrôle qui contribueront réellement à l'amélioration de la surveillance, de la prévention et du contrôle de la fièvre jaune dans le pays ${ }^{2} 0$.

Des initiatives comme celles-ci soutiennent la production de connaissances pour l'amélioration de la gestion et de l'opérationnalisation du système de surveillance épidémiologique. Ainsi, il convient d'accroître la compréhension du processus de dissémination du virus de la fièvre jaune, en tenant compte des facteurs qui favorisent le risque d'exposition, les mécanismes d'assimilation et la perception précoce de la diffusion virale des primates et des vecteurs, et aussi qui peuvent catégoriser et différencier la menace entre les zones prédisposées et vulnérables. Surtout lorsque les civils ne sont pas vaccinés ${ }^{1} 5$.

Cependant, il y a encore de graves difficultés dans les municipalités pour assurer l'avancée complète d'un PVCFA notoire et efficace, y compris des fonds d'immobilisations insuffisants, des problèmes de formation en laboratoire face aux demandes nécessaires et des actions de vigile l'utilisation épidémiologique des ressources en raison de la mauvaise gestion par les politiciens locaux. En plus des ressources financières toujours rares pour le secteur de la santé ${ }^{1} 7$.

Par conséquent, on s'attend à ce que les outils déjà en vigueur puissent soutenir l'expansion et la formation du cadre des professionnels de la santé qui sont prêts à accepter les défis de l'opérationnalisation et de la restructuration de la surveillance épidémiologique de la fièvre jaune dans le outils de soutien pour une meilleure exécution de sa fonction en tant qu'instrument de changement, d'intervention et d'incitation à la santé.

\section{CONCLUSION}

Avec l'urbanisation des centres ruraux, la possibilité d'émergence de certaines maladies, comme la fièvre jaune, s'accroît, ce qui aurait de graves conséquences pour 
la santé publique, en particulier dans les régions où le taux de couverture vaccinale est faible.

La modification de l'environnement causée par l'activité forestière, l'ouverture des routes, l'agriculture, entre autres, exposer accidentellement les individus non vaccinés aux vecteurs d'invertébrés sauvages, étant l'une des principales formes de transmission du mazela en Amazonie brésilienne. Ce fait a été constitué au fil des ans comme une justification pertinente pour les nombreux cas de mortalité qui se sont produits dans les États de l'Amazonie juridique. Jusqu'au début de 2017, au Brésil, des cas épisodiques se sont produits dans des États situés à l'extérieur de cette zone ont été identifiés. Cependant, les premiers mois de 2017 ont révélé la nécessité d'intensifier la surveillance, étant donné que l'expansion de l'épidémie implique d'autres États que le Minas Gerais, tels que Gois, Sao Paulo et Mato Grosso, en plus des États amazoniens. Ce besoin s'explique par le fait qu'un patient virulent d'une zone endémique de fièvre jaune sauvage peut, associé aux fortes densités d'Aedes aegypti, constituer un stimulant potentiel pour la réurbanisation de la maladie au Brésil. Cette déclaration est renforcée par des études en cours, qui indiquent la présence d'Aedes aegypti dans environ 21 États brésiliens7, 'e qui renforce la nécessité d'une surveillance.

De ce point de vue, la nécessité d'un PVCFA efficace se pose, afin qu'il puisse, en fait, exercer un contrôle sur les foyers possibles de la maladie et, en outre, atteindre par l'éducation à la discipline de santé et la formation des professionnels pour maintenir le risque de épidémie loin de la population urbaine.

\section{RÉFÉRENCES}

1. VERONESI, R - Tratado de infectologia. São Paulo: Editora Atheneu, 1996.

2. COSTA, GZA; ROMANO, APM; ELKHOURY, ANM; FLANNRY, B - Evolução histórica da vigilância epidemiológica e do controle da febre amarela no Brasil. In: Rev Pan-Amaz Saude. 2011. Disponível em: http://scielo.iec.pa.gov.br/scielo.php?pid=S217662232011000100002\&script=sci_arttext\&tIng=en. Acessado em: 01 dez. 2016. 
3. MONATH, T.P - Yellow fever: an update. In: Lancet v.1, 2001, p.11-20.

4. QUARESMA, JAS - Apoptose e citocinas mediadoras da lesão hepática na febre amarela: subsídios para o redirecionamento dos mecanismos de lesão e suas consequências. 2003. 245-256f. Tese (Doutorado em Patologia Clínica) Faculdade de Medicina, Universidade de São Paulo, São Paulo, 2003.

5. LEÃO, RNQ - Doenças Infecciosas e parasitárias: enfoque amazônico. Belém: CEJUP, 1997.

6. GALLE, RP; HOFMANN, WJ; WALCZAK, H; SCHALLER, H; OTTO, GSW; KRAMMER, PH.; RUNKEL, L - Envolvement of the CD95 (APO-1/Fas) receptor and ligand in liver damage. In: J. Exp. Med. v.182, p.1223-1230, 1998.

7. MASSAD, E; COUTINHO, FAB; BURATTINI, MN; LOPES, LF - The risk of yellow fever in a dengue-infested area. In: Trans. R. Soc. Trop. Med. Hyg. v.95, 2001, p.570-574.

8. FRANCO, O - A história da febre amarela no Brasil. Rio de Janeiro: Ministério da Saúde; 2009.

9. LACERDA, A - Em busca de um tempo perdido: fontes visuais para a história da vacina contra a febre amarela. In: BENCHIMOL, J - Febre amarela: a doença e a vacina, uma história inacabada. Rio de Janeiro: FIOCRUZ; 2010.

10. PORTO, M - Marcos técnicos e legais para a descentralização do controle de endemias. Brasília: Ministério da Saúde, 2014.

11. BENCHIMOL, J - Febre amarela: a doença e a vacina, uma história inacabada. Rio de Janeiro: FIOCRUZ, 2010.

12. BRANCO, J - Vigilância da febre amarela no Brasil. Rio de Janeiro: Fundação Oswaldo Cruz, 2008.

13. CALHEIROS, L - A febre amarela no Brasil. Rio de Janeiro: Fundação Oswaldo Cruz, 2008.

14. COSTA, GZA - Estudo das características epidemiológicas da febre amarela no Brasil, nas áreas fora da Amazônia Legal, período de 1999-2003 [dissertação]. Belém: Universidade Federal do Pará, 2005.

15. RIBAS, E - O mosquito como agente de propagação da febre amarela. Porto Alegre: Artmed, 2009. 
16. SILVA, LM - Descentralização do controle de endemias. Brasília: Ministério da Saúde, 2011.

17. FARIA, GS - Endemias Rurais: métodos de trabalho adotados pelo DNERu. Rio de Janeiro: Ministério da Saúde, 2006.

18. HOMMA, A; CUNHA, J - Febre Amarela e Dengue. Rio de Janeiro: Fundação Oswaldo Cruz. 2007.

19. CAUSEY, C - Implantação dos estudos sobre arbovírus na região amazônica. 13 ed. Belém: Fundação Serviços de Saúde Pública, 2005.

20.BRASIL. Ministério da Saúde - Guia de vigilância epidemiológica. 15 ed. Brasília: Fundação Nacional de Saúde, 2012.

21. BRASIL. Ministério da Saúde - Plano de Intensificação das Ações de Prevenção e Controle da Febre Amarela no Brasil. Brasília: Ministério da Saúde, 2008.

22. DEMO, P - Metodologia do conhecimento científico. 5 ed. São Paulo: Atlas, 2009.

23. COSTA, GZA; ROMANO, APM; ELKHOURY, ANM; FLANNRY, B - Evolução histórica da vigilância epidemiológica e do controle da febre amarela no Brasil. In: Rev Pan-Amaz Saude. 2011. Disponível em: http://scielo.iec.pa.gov.br/scielo.php?pid=S2176-

62232011000100002\&script=sci_arttext\&tIng=en. Acessado em: 29 nov. 2016.

24. LIMA-CAMARA, T.N. Arboviroses emergentes e novos desafios para a saúde pública no Brasil. In: Rev. Saúde Pública. Disponível em: http://www.scielo.br/scielo.php?pid=S0034-

$89102016000100602 \&$ script=sci_arttext\&tIng=pt. Acessado em: 25 out. 2019.

25.LOPES, N; NOZAWA, C; LINHARES, R.E.C. Características gerais e epidemiologia dos arbovírus emergentes no Brasil. In: Revista Pan-Amazônica de Saúde. Disponível em:

http://scielo.iec.gov.br/scielo.php?script=sci_arttext\&pid=S217662232014000300007 . Acessado em: 25 out. 2019.

26.CAMPOS, F.S. Replicação Viral. 2018. Disponível em: http://www.ufrgs.br/labvir/material/replicacao_med.pdfAcessado em: 25 out. 2019. 
27. BRASIL. Centro de Vigilância Epidemiológica. Sobre a Febre Amarela. 2018. Disponível em: http://www.saude.sp.gov.br/resources/cve-centro-de-vigilanciaepidemiologica/areas-de-vigilancia/doencas-de-transmissao-por-vetores-ezoonoses/famarela.html. Acessado em: 25 out. 2019.

28. GOMES, A.C; et al. Ecologia de Haemagogus e Sabethes (Diptera: Culicidae) em áreas epizoóticas do vírus da febre amarela, Rio Grande do Sul, Brasil. In: Epidemiologia e Serviços de Saúde. 2010. Disponível em: http://scielo.iec.gov.br/scielo.php?script=sci_arttext\&pid=S167949742010000200003. Acessado em: 25 out. 2019.

29. SUCCI, R.C.M; FARHAT, C.K. Vacinação em situações especiais. In: Jornal de Pediatria. 2006. Disponível em: http://www.scielo.br/scielo.php?script=sci_arttext\&pid=S002175572006000400011. Acessado em: 25 out. 2019.

30.CAMPOS, A.C.T; MATTOS, S.V.M. Avaliação de requisitos referentes à implantação do sistema de gestão da qualidade nos Laboratórios Centrais de Saúde Pública. In: Revista do Instituto Adolfo Lutz. 2009. Disponível em: http://periodicos.ses.sp.bvs.br/scielo.php?script=sci_arttext\&pid=S007398552009000300019\&lng=pt\&nrm=iso. Acessado em: 25 out. 2019.

Soumis : août 2019.

Approuvé : novembre 2019. 